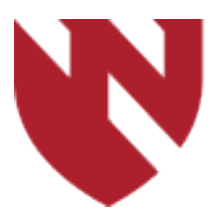

December 2019

\title{
Virtual Reality (VR) to Improve the Experience of the Mohs \\ Patient: A Prospective Interventional Study
}

\author{
Shauna Higgins \\ University of Nebraska Medical Center \\ Shera Feinstein \\ University of Nebraska Medical Center \\ Makenzie Hawkins \\ University of Nebraska Medical Center \\ Myles Cockburn \\ University of Nebraska Medical Center \\ Ashley Wysong \\ University of Nebraska Medical Center
}

Tell us how you used this information in this short survey.

Follow this and additional works at: https://digitalcommons.unmc.edu/gmerj

Part of the Higher Education Commons, and the Medicine and Health Sciences Commons

\section{Recommended Citation}

Higgins, S., Feinstein, S., Hawkins, M., Cockburn, M., , Wysong, A. Virtual Reality (VR) to Improve the Experience of the Mohs Patient: A Prospective Interventional Study. Graduate Medical Education Research Journal. 2019 Dec 13; 1(1).

https://digitalcommons.unmc.edu/gmerj/vol1/iss1/12

This Conference Proceeding is brought to you for free and open access by DigitalCommons@UNMC. It has been accepted for inclusion in Graduate Medical Education Research Journal by an authorized editor of DigitalCommons@UNMC.For more information, please contact digitalcommons@unmc.edu. 


\section{Virtual Reality (VR) to Improve the Experience of the Mohs Patient: A Prospective Interventional Study}

\section{Creative Commons License}

\section{(c) (1) $\Theta(9$}

This work is licensed under a Creative Commons Attribution-Noncommercial-No Derivative Works 4.0 License. 


\section{Virtual Reality (Vr) to Improve the Experience of the Mohs Patient - A Prospective Interventional Study Shauna Higgins, Shera Feinstein, Makenzie Hawkins, Myles Cockburn, Ashley Wysong}

Mentor: Ashley Wysong

Program: Dermatology

Background: Non-melanoma skin cancer (NMSC) is the most common cancer in the US with significant quality of life (QOL) impact.

Objective: To assess the utility of a highly immersive virtual reality (VR) experience in the context of outpatient skin cancer surgery as a means to minimize patient reported feelings of anxiety or pain. We also sought to assess the effects on patient reported overall satisfaction.
Materials \& Methods: Patients completed a pre-VR experience survey after completion of their first Mohs surgery layer, followed by a 10-minute virtual reality experience, and a post-VR experience survey. Differences in the pre-VR survey and post-VR survey were compared using the Chi-square test. The anxiety scores were compared using a t test.

Results: In all but two questions, there was a trend towards improvement of the anxiety related sensations after completion of the VR experience. There were statistically significant differences for four questions: "Are you currently feeling unable to relax" $(p=0.0013)$, "are you currently feeling fear of the worst happening" ( $p<0.0001)$, "are you currently feeling terrified or afraid" $(p=0.0046)$, and "are you currently feeling nervous" $(\mathrm{p}<0.0001)$.

Conclusion: VR experiences during the Mohs surgical day significantly improved measures of anxiety and patient satisfaction.

https://doi.org/10.32873/unmc.dc.gmerj.1.1.012

\section{A Performance Improvement Project in Antibiotic Administration for Open Fractures Benjamin Ogden, Christopher Deans, Matthew Mormino, Michaela McCarthy, Elizabeth Lyden, Justin Siebler}

Mentor: Justin Siebler

Program: Orthopaedic Surgery

Purpose: To evaluate the effects of a trauma performance improvement project involving standardized protocols for administration of antibiotics in open fractures at a level one trauma center. This study specifically evaluated the protocol's efficacy for improving the timing of delivery, appropriate therapy administration, and sought to identify factors that lead to delay of antibiotic delivery.

Methods: Retrospective comparative cohort study comparing patients with open fractures treated at our hospital between January 2013 and September 2015 (Group 1) and between April 2016 and June 2017 (Group 2). Group 1 was prior to and Group 2 was after implementation of the performance improvement project.

Results: Group 1 consisted of 79 patients and Group 2 consisted of 80 patients with open fractures. Each group was statistically similar in patient and injury factors. Group 1 received antibiotics at an average of 97 minutes after arrival to our hospital while Group 2 patients received them at an average of 46 minutes $(p<0.0001)$. The average time from admission to initial evaluation improved from 10 minutes to 3 minutes ( $p<0.0001$ ). Average time from evaluation to antibiotic order placement improved from 77 minutes and 26 minutes $(\mathrm{p}<0.0001)$. Average time from order entry to antibiotic administration showed no significant difference (12 minutes vs 15 minutes, $\mathrm{p}=0.25)$. Thirty-four percent (27/79) of Group 1 patients and 84\% (67/80) of Group 2 patients received antibiotics within 1 hour of admission $(\mathrm{p}<0.0001)$, while $91 \%$ and $99 \%$ received antibiotics within 3 hours, respectively $(\mathrm{p}=0.03)$.

Conclusions: A multifaceted performance improvement protocol was highly effective for producing a more coordinated, efficient, and timely process for administration of antibiotics to patients with open fractures at our hospital.

https://doi.org/10.32873/unmc.dc.gmerj.1.1.013

\section{The Effects of Stiff Stent-Grafts on Segmental Aortic Stiffness and Left Ventricular Mass: A Pilot Swine Model Study Paul Aylward, Alexey Kamenskiy, Jason MacTaggart}

Mentor: Jason MacTaggart

Program: General Surgery

Introduction: Endovascular stent-grafting is a first-line therapy for many aortic pathologies, but stent-grafts are constructed using noncompliant materials that stiffen a segment of the aorta. This diminishes the Windkessel function, reducing the ability to dampen peak systolic pressures and provide aortic wall recoil, and may lead to left ventricular (LV) hypertrophy. We tested this hypothesis in a swine model.

Methods: CTA imaging was performed to assess aortic geometry, and a commercially available stent-graft was implanted into the thoracic descending aorta of a juvenile swine. Pressure waveforms were obtained in the aorta, coronary, carotid, and renal arteries before and after stent-graft placement. After 8 weeks, CTA and pressure measurements were repeated, and the heart and aorta were histologically and mechanically evaluated. An age-matched swine was used as a control.

Results: After stent-graft placement, the pulse wave velocity increased 2.5-fold, and pressure waveforms changed their shape. At 8 weeks, the stent-graft animal mean arterial pressure increased $25 \mathrm{mmHg}$, aortic wall recoil reduced 1.2-2.0-fold proximal to the stent-graft, and was eliminated within the stent-grafted segment. The aorta remodeled to a larger diameter, and became stiffer than control, proximal to and behind the stentgraft. LV mass increased $48 \%$ in the stentgraft animal, but only $28 \%$ in the control.
Cardiac and aortic histology demonstrated signs of fibrosis, increased cellularity, and increased MMP activity in the stent-graft animal.

Conclusions: Stiff aortic stent-grafts reduce the Windkessel effect, which may lead to higher pressures, altered pressure waveforms, an inflammatory response, stiffening of the ascending aorta, and LV hypertrophy, which warrants further investigation.

https://doi.org/10.32873/unmc.dc.gmerj.1.1.014 